\title{
EQUILIBRIUM OF TRUSS AND BEAM STRUCTURES OF INELASTIC MATERIALS
}

\author{
Lars German HAGSTEN \\ Aarhus University, Aarhus School of Engineering/Department of Engineering, \\ Inge Lehmanns gade 10, DK-8000 Aarhus C, Denmark
}

Received 06 May 2016; accepted 18 Aug 2016

\begin{abstract}
A physically based method for the determination of equilibrium for structures with inelastic response is described. The method is based on minimisation of the potential energy. For structures with inelastic response, some of the applied energy is converted to non-mechanical energy. This part of the energy is dissipated. According to the conservation of energy the dissipated energy must simultaneously be subtracted the mechanical energy in order to determine the change of the potential energy. Changes of the strains in the structure, from non-static conditions, such as thermal deformations and shrinkage, as well as plastic strains from previous load scenarios, will also change the potential energy. The method is also capable of taken these effects into account. Three examples are included in order to support the physical understanding, and to illustrate the procedure for the application of the method. Information regarding the necessary ductility of the individual parts forming the complete structure is achieved as outcome of the analysis.
\end{abstract}

Keywords: equilibrium, energy methods, dissipation, potential energy, ductility, structures, inelastic materials.

\section{Introduction}

The majority of structures are constructed of materials with a certain degree of ductility. These structures behave inelastic at high load levels. For statically indeterminate structures the maximum load capacity is typically somewhat higher than the maximum load level at which all parts behave elastically. Utilization of the extra capacity requires knowledge of the necessary ductility of the individual parts of the structure. This is illustrated by the two static systems shown in Figure 1. In Figure 1a is shown a two times statically indeterminate truss structure subjected by a vertical load, $P$. In Figure $1 \mathrm{~b}$ is shown a two times indeterminate frame structure subjected by a horizontal load, $P$. By modelling the material as ideal plastic the capacity is determined by either the use of statically admissible stress distribution (according to the lower bound theorem) or by creating a mechanism (according to the upper bound theorem), see Beedle (1957), Bruneau et al. (2011), Drucker et al. (1952), Marti (2013) and Nielsen and Hoang (2011).

In both cases the plastic capacity is higher than the elastic capacity. The truss structure in Figure 1a has a plastic capacity which is $28 \%$ higher than the elastic capacity, corresponding to first yielding. The frame structure in Figure $1 \mathrm{~b}$ has a plastic capacity which is $18 \%$ higher than the elastic capacity. The internal forces in the individual members in the frame structure are varying. Utilization of the plastic capacity requires therefor, that the yield zone has a certain length and not just points as indicated in Figure 1b. The fact that the yield zones must have a certain length combined with the varying internal forces therefore requires materials which exhibits strain hardening in order to achieve a capacity which is higher than the elastic capacity. For this reason there will also be a small difference between the elastic and plastic analysis in terms of the maximum moment in the most heavily affected sections.

The question is which level of ductility is needed in order to reach the plastic capacity. In order to address this question knowledge of the strains or curvatures in the individual parts of the structure is required.

Utilization of the post-yield behaviour plays an important role in the design of reinforced concrete structures, see Einpaul et al. (2015), Fisker et al. (2016), Lee et al. (2011), Marti (2013), Muttoni et al. (2015), Nielsen and Hoang (2011), Simões et al. (2016), Ruiz et al. (2013) as well as in the design of steel structures, see Berman and Bruneau (2003), Bruneau et al. (2011), Montuori et al. (2014, 2015), Nastri et al. (2015), Peng et al. (2013), Qu and Bruneau (2015), Sahoo and Chao (2010).

Corresponding author: L. G. Hagsten

E-mail:lgh@ase.au.dk 


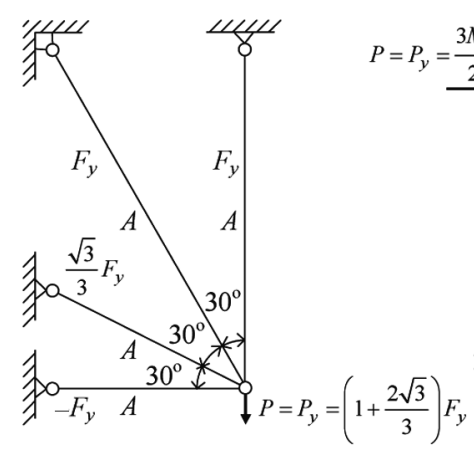

a)

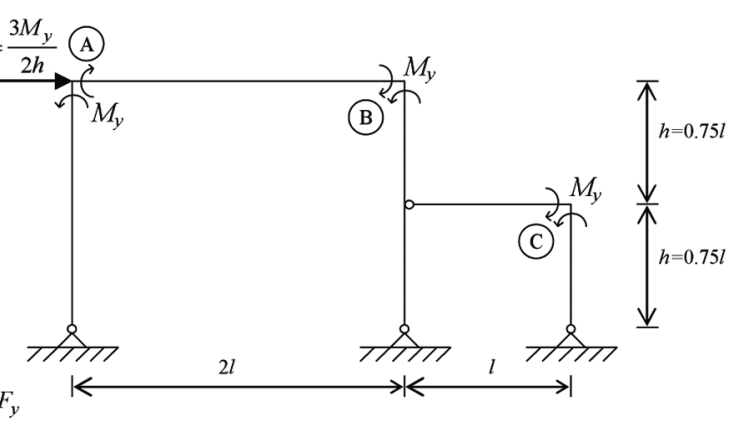

b)

Fig. 1. Two examples of structures constructed of ideal plastic materials: a) truss structure, b) frame structure

The presented method also takes non-statical deformations from shrinkage and thermal deformations into account. Shrinkage and thermal deformations are important in the assessment of the structural behaviour, see Balázs et al. (2013).

Equilibrium for a structure can be determined by minimisation of the potential energy, see, for instance, Feynman et al. (2011). Hereby both the static and geometrical conditions will be met. By means of the principle of virtual work, this can for statically indeterminate structures, be shown to be identical to the minimization of the strain energy, see Castigliano (1879) and Castigliano et al. (1966). This approach has however, traditionally, been applied only to structures made of elastic materials. In this paper it is shown how the principles of minimising the potential energy can be applied, also for structures made from inelastic materials. Based on the principle of conservation of energy, see Helmholtz (1847), the stored heat energy must therefore be subtracted the energy of the structure. The stored heat energy represent an increase in the kinetic energy of the molecules and this kind of energy will in the following be named dissipated energy. Since the sum of the kinetic and potential energy is constant, the dissipated energy must simultaneously be subtracted in order to determine the change in mechanical which is identical to the potential energy. Therefore, regarding the change of potential energy in the materials, only the change in stored elastic energy is included.

The basic analytical expression for the determination of the potential energy is the same whether the stresses is constant or variable in the individual parts of the structure. For structures with no variation of stresses in the individual members, as for instance truss structures, the expression that describes the minimum of the potential energy can be set up as a function of the strain in the material. The expression on this form is generally applicable, and is thus basically identical to the expression for structures of elastic materials. The difference between structures of elastic and inelastic materials appears therefore only to consist in the expression for the corresponding strains.
Using the above described method a full physical description of the determination of equilibrium for structures of inelastic materials is obtained.

The principles presented are illustrated by means of both a statically indeterminate truss structure and a statically indeterminate frame structure. The principles can as well be used for combined truss and frame structure, see Wongpakdee et al. (2014). Considerations set out in the article relate to the behaviour of statically indeterminate structures constructed of materials with arbitrary constitutive relation, and are exemplified by materials with a bilinear constitutive relation. A survey of the developments in the structural analysis of steel frame structures subjected to static loading is given in Nethercot (2000).

The problem has to some extent been dealt with by introducing the non-physical concept of complementary elastic energy and by applying the principle of virtual work (Engesser 1889). An overview has been presented by Santos (2011).

\section{Theory}

As an outset for the description, the potential energy has been chosen to be zero for the unloaded structure.

When determining the change of potential energy for a structure constructed of elastic materials subjected by loads, the change of potential energy is given by the sum of a contribution from the external loads, $\Pi_{P}$, and a contribution from the elastic energy stored in the material, $\Pi_{\text {elastic }}$. By removing the load from the system the potential energy will again be zero.

On the contrary, by first applying a load to a structure constructed of inelastic materials, and afterwards removing the load, the potential energy seems to be smaller than zero when only taking $\Pi_{P}$ and $\Pi_{\text {elastic }}$ into account. Some of the energy, $\Delta w$, has dissipated.

According to the conservation of energy the dissipated energy, $\Delta w$, must be a part of the energy balance in order to determine the change of the potential energy:

$$
\Pi-\Delta w=\Pi_{P}+\Pi_{\text {elastic }} .
$$


Equation (1) can be rewritten:

$$
\Pi=\Pi_{P}+\Pi_{\text {elastic }}+\Delta w .
$$

The state of equilibrium is found for stationary values of the potential energy.

A simple bar subjected to a load, $P$, is considered. The force-displacement relationship illustrated in Figure $2 \mathrm{a}$ is used.

A load greater than the yield load $\left(P>f_{y} A\right)$ is applied, and the value of $y$ which provides the state of equilibrium is deduced, i.e. the value of $y$ which provides minimum of the potential energy, where $y$ is gradually increased from zero until equilibrium is achieved.

The change in potential energy is illustrated by the thick line in Figure $2 \mathrm{~b}$. The bar behaves elastically up to $y=y_{\mathrm{A}}$. Hence there is no dissipation up to $y=y_{\mathrm{A}}$. At $y=y_{\mathrm{A}}$ the limit of proportionality is reached. The potential energy is given by $\Pi_{\mathrm{A}}$. For increased elongation a part of the energy is dissipated. At the deformation $y=y_{\mathrm{B}}$ the total loss of energy is given by $\Pi_{\mathrm{B}}{ }^{\prime}$. As mentioned a part of this loss is dissipated energy, and this part must be subtracted simultaneously from the structure in order to estimate the change of the potential energy. This subtracted part is seen to be given by $\Pi_{\mathrm{B}}-\Pi_{\mathrm{B}}{ }^{`}$, whereby the loss in potential energy is given by $\Pi_{\mathrm{B}} . \Pi_{\mathrm{B}}$ is in the example also identical to the minimum of the potential energy, $\Pi_{\mathrm{min}}$. The corresponding extension, $y_{\mathrm{B}}$ is identical to the position of equilibrium. At a further increase of $y$, the loss of potential energy increases further, but the increase in dissipated energy is seen to be increased even more, whereby $\Pi_{\mathrm{B}}$ is the minimum value of the potential energy. The two shaded areas do both represent the dissipated energy.

The problem can also be analysed in another way. The state of equilibrium is given by a deformation which is greater than $y_{\mathrm{A}}$. The bar is then unloaded $(P=0)$. Next, the bar is again loaded to $P_{u}$. During this loading the bar behaves elastically (with stiffness $k_{3}$ ). The state of equilibrium can then be determined by a classical energy consideration by determining a stationary state for the sum of $\Pi_{P}$ and $\Pi_{\text {elastic }}$, that is $\frac{d \Pi}{d(\Delta y)}=0 \Rightarrow \Delta y=\frac{P_{u}}{k_{3}}$ $\Rightarrow y=y_{\text {plastic }}+\Delta y$. The difference between this analysis and the previous analysis is that, for the later analysis, the deformation is split up in two contributions and $y_{\text {plastic }}$, which is related to the dissipation, will not be a result of the analysis. This is also the reason why it is not possible to use the later analysis in general as the magnitude of the dissipation is not known a priory. Therefor it is necessary to use the unloaded/undeformed structure as outset for the analysis and have the dissipation as an unknown quantity which is linked to $\Pi_{P}$ and $\Pi_{\text {elastic }}$ through the geometric quantities.

For statically indeterminate structures, the same analogy with respect to unloading/reloading can be used. Thereby the significance of the dissipation will be clarified. During reloading the change of the stress state in the individual members will be purely elastic. Also in this case the magnitude of the dissipation will be unknown. The unknown magnitude of the dissipation is the reason why the expression for the dissipation must be a part of the expressions and linked to $\Pi_{P}$ and $\Pi_{\text {elastic }}$ through the geometric quantities. For a m-times statically indeterminate structure equilibrium is found for:

$$
\frac{d \Pi}{d X_{1}}=0, \frac{d \Pi}{d X_{2}}=0, \ldots ., \frac{d \Pi}{d X_{m}}=0,
$$

where $X_{i}$ are the redundant variables.

In the following the potential energy is expressed for some statically indeterminate structures. Starting by analysing truss structures which are characterized by constant internal forces in the individual members. Subsequently a frame structures which, on the contrary, in general have variable distribution of internal forces is analysed.

\subsection{Constant internal stresses}

A method for structures with constant internal forces is found through an analysed example. Afterwards the

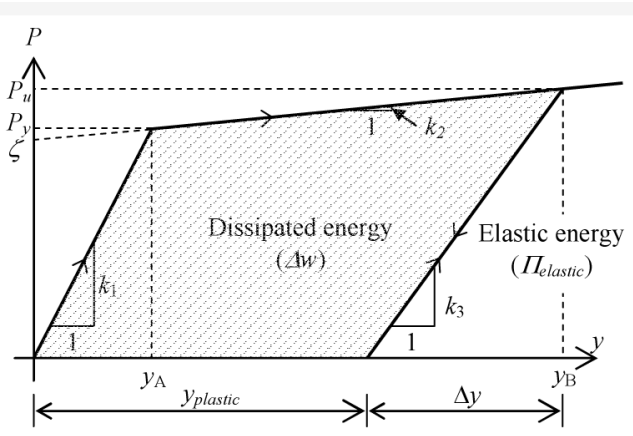

a)

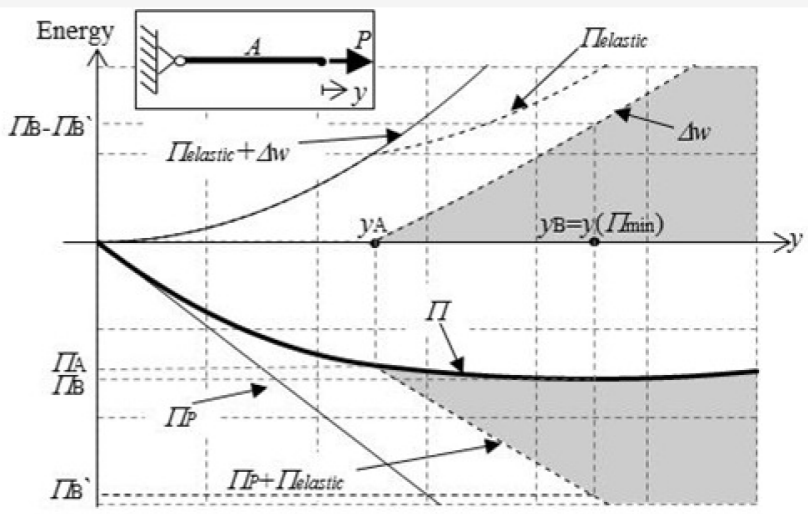

b)

Fig. 2. a) Bilinear stress-strain relation. Material with inelastic stress-strain behaviour for $y>y_{y}=y_{\mathrm{A}}$. At unloading for $y>y_{\mathrm{A}}$ the stiffness is given by $k_{3}$; b) Different contributions to the energy as function of the elongation of the tension bar 


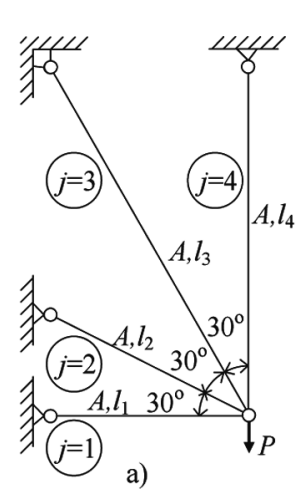

a)

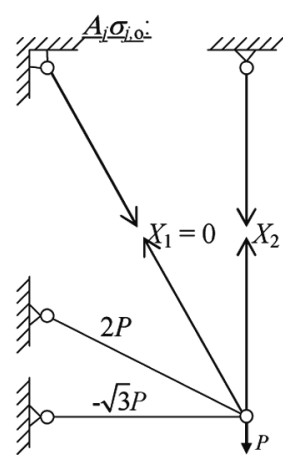

b)

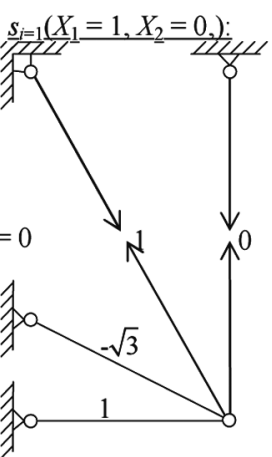

c)

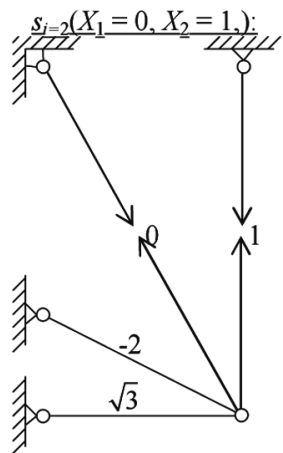

d)

Fig. 3. a) Structure; b) Statically determinate basic system; c) and d) $s_{1}$ and $s_{2}$ for $X_{1}=1$, respectively $X_{2}=1$

method is generalised. First the two times statically indeterminate truss structure shown in Figure 3a is analysed. All bars are assumed to have the same cross-sectional area, A. Problems related to instability are disregarded.

The stress distribution is analysed using the principle of the force method. The stress distribution is therefore analysed by defining a statically determinate basic system. This has been chosen to consist of the bars 1 and 2 , see Figure $3 b$. This statically determinate basic system is loaded by the external load. Accordingly, the redundant is expressed by the stresses in the bars $3\left(X_{1}\right)$ and $4\left(X_{2}\right)$, see Figures $3 \mathrm{c}$ and $3 \mathrm{~d}$.

The stresses in the individual bars can thus be expressed as linear combinations of $P, X_{1}$ and $X_{2}$.

$$
\begin{aligned}
& \sigma_{1}=\frac{-\sqrt{3} P}{A}+1 \cdot X_{1}+\sqrt{3} \cdot X_{2}, \\
& \sigma_{2}=\frac{2 P}{A}-\sqrt{3} \cdot X_{1}-2 \cdot X_{2}, \quad \sigma_{3}=X_{1}, \quad \sigma_{4}=X_{2} .
\end{aligned}
$$

Any combination of $X_{1}$ and $X_{2}$ will thus be in equilibrium with the external load. The stresses from the redundant variables can be considered as states of eigenstresses. The question is which values of $X_{1}$ and $X_{2}$ will be the actually occurring values. That means which values of $X_{1}$ and $X_{2}$ will also fulfil both the geometrical and statical conditions.

The actual values of $X_{1}$ and $X_{2}$ are determined by requiring the potential energy to be stationary.

A situation in which the load has a magnitude so that yielding occurs in 3 and 4 while bar 1 and bar 2 behaves elastically is examined. The stress-strain relation for the material is shown in Figure 4.

The individual components in Eqn (2) are determined for the static system shown in Figure 3.

$$
\begin{gathered}
\Pi_{\text {elastic }}=\frac{1}{2} \frac{\sigma_{1}^{2}}{E_{1}} A l_{1}+\frac{1}{2} \frac{\sigma_{2}^{2}}{E_{1}} A l_{2}+\frac{1}{2} \frac{\sigma_{3}{ }^{2}}{E_{2}} A l_{3}+\frac{1}{2} \frac{\sigma_{4}{ }^{2}}{E_{2}} A l_{4} ; \\
\Pi_{P}=-P y .
\end{gathered}
$$

Here $P$ is the external load and $y$ is the displacement of this load. In the state of equilibrium both the statically and geometrical conditions are met, which means that the applied loads are in equilibrium with the internal stresses, and the deformation at the applied load is compatible with the strains in the structure. Thereby, using the principle of virtual work in the particular case with real static and geometric quantities, $\Pi_{P}=-P y$ can be replaced by $\Pi_{P}=-\sum_{i=1}^{4} \sigma_{i} \varepsilon_{i} A_{i} l_{i}$ for the statically indeterminate structure.

Dissipation takes places in bar 3 and bar 4 . This amounts to:

$$
\begin{array}{r}
\Delta w=\left(\sigma_{3} \varepsilon_{3}-\frac{1}{2} \zeta \varepsilon_{y}-\frac{1}{2}\left(\sigma_{3}-\zeta\right) \varepsilon_{3}-\frac{1}{2} \frac{\sigma_{3}^{2}}{E_{2}}\right) A l_{3}+ \\
\left(\sigma_{4} \varepsilon_{4}-\frac{1}{2} \zeta \varepsilon_{y}-\frac{1}{2}\left(\sigma_{4}-\zeta\right) \varepsilon_{4}-\frac{1}{2} \frac{\sigma_{4}^{2}}{E_{2}}\right) A l_{4} .
\end{array}
$$

Constitutive equation for $\varepsilon_{j}>\varepsilon_{y}$ :

$$
\varepsilon_{j}=\frac{\sigma_{j}-\zeta}{E_{s h}} .
$$

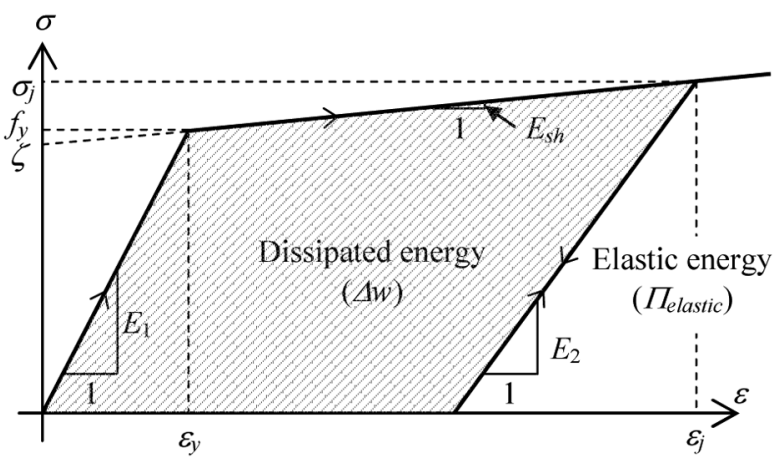

Fig. 4. Bilinear stress-strain relation. Material with inelastic stress-strain behaviour for $\varepsilon_{j}>\varepsilon_{y}$. At unloading for $\varepsilon_{j}>\varepsilon_{y}$ stiffness is given by $E_{2}$ 
By inserting Eqn (8) in Eqn (7):

$$
\begin{array}{r}
\Delta w=\left(\frac{\sigma_{3}\left(\sigma_{3}-\zeta\right)}{E_{s h}}-\frac{1}{2} \zeta \frac{f_{y}}{E_{1}}-\frac{1}{2} \frac{\left(\sigma_{3}-\zeta\right)^{2}}{E_{s h}}-\frac{1}{2} \frac{\sigma_{3}^{2}}{E_{2}}\right) A l_{3}+ \\
\left(\frac{\sigma_{4}\left(\sigma_{4}-\zeta\right)}{E_{s h}}-\frac{1}{2} \zeta \frac{f_{y}}{E_{1}}-\frac{1}{2} \frac{\left(\sigma_{4}-\zeta\right)^{2}}{E_{s h}}-\frac{1}{2} \frac{\sigma_{4}^{2}}{E_{2}}\right) A l_{4} .
\end{array}
$$

By solving $\frac{d \Pi}{d X_{1}}=0, \frac{d \Pi}{d X_{2}}=0$ the values of $X_{1}$ and $X_{2}$ which fulfil both the geometrical and statical conditions is found. $\Pi$ is found by Eqn (2) using the expressions for $\Pi_{P}, \Pi_{\text {elastic }}$ and $\Delta w$.

It is generally assumed that the load is increased continuously, and that no single part is relieved.

In general it applies for a truss structure which is mtimes statically indeterminate that the stress in the $j^{\text {th }}$ bar may be expressed by:

$$
\sigma_{j}=\sigma_{j, 0}+s_{j, 1} X_{1}+s_{j, 2} X_{2}+\ldots+s_{j, m} X_{m},
$$

where $\sigma_{j, 0}$ is the stress in the $j^{\text {th }}$ bar produced by the known external loads acting on the statically determined truss obtained by having all $X$ s equal to zero. $s_{j}$ is found by setting the corresponding unit force, $X=1$ and the remaining $X$ s and the external loads equal to zero in the statical determinate truss. $s_{j} X_{m}$ is the stress produced in the $j^{\text {th }}$ bar by the redundant $X_{m}$.

It is assumed that all bars exhibit an inelastic relationship between strains and stress for $\varepsilon_{i}>\varepsilon_{y}$, see Figure 4.

The contribution from the external loads in general:

$$
\Pi_{P}=-\sum_{k=1} \bar{P}_{k} \cdot \bar{y}_{k}
$$

where $P$ is the external load and y is the displacement of the load. As in the example above $E_{p o t, P}=-\Sigma P-y$ can be replaced by $E_{p o t, P}=-\Sigma \sigma_{j} \varepsilon_{j} A_{j} l_{j}$ for a statically indeterminate structure by using the principle of virtual work in the particular case with real static and geometric quantities. $A_{j}$ and $l_{j}$ is respectively the cross sectional area and the length of the $j^{\text {th }}$ bar.

The elastic energy stored in the structure:

$$
\Pi_{\text {elastic }}=\sum_{j=1}^{n} \frac{1}{2} \frac{\sigma_{j}^{2}}{E_{2}} \cdot A_{j} l_{j},
$$

where $n$ is the numbers of elements.

The dissipated energy, which is identical to the loss of potential energy can be written as:

$$
\Delta w=\sum_{j=1}^{n}\left(\begin{array}{c}
\sigma_{j} \varepsilon_{j} \cdot A_{j} l_{j}-\frac{1}{2} \zeta \cdot \varepsilon_{y} \cdot A_{j} l_{j} \\
-\frac{1}{2}\left(\sigma_{j}-\zeta\right) \cdot \varepsilon_{j} \cdot A_{j} l_{j}-\frac{1}{2} \frac{\sigma_{j}^{2}}{E_{2}} \cdot A_{j} l_{j}
\end{array}\right) .
$$

Changes of strains in the structure, from non-static conditions, such as thermal deformations and shrinkage will also change the potential energy in the individual members by $\sigma_{j} \varepsilon_{j, 0} A_{j} l_{j}$, where $\varepsilon_{j, 0}$ can represent both thermal deformations ( $\alpha \Delta t)$, shrinkage or plastic strains from previous load scenarios (positive as elongations). Eqns (11), (12) and (13) are inserted into Eqn (2). Some part can be assembled/respectively be deleted. Please note that the two parts containing $E_{2}$ are equal to each other, whereby $E_{2}$ vanish from the equation. The value of $E_{2}$ is thus irrelevant, and need not to be identical to $E_{1}$ or any other specific value:

$$
\Pi=-\sum_{j=1}^{n}\left(\frac{1}{2} \zeta \cdot \varepsilon_{y} \cdot A_{j} l_{j}+\frac{1}{2}\left(\sigma_{j}-\zeta\right) \cdot \varepsilon_{j} \cdot A_{j} l_{j}+\sigma_{j} \varepsilon_{j, 0} A_{j} l_{j}\right) .
$$

Stationary values of Eqn (14) give the equilibrium of the structures. For structures with no variation of stresses in the individual members, the derivatives of Eqn (14) can be found straight forward. The derivative that gives the state of equilibrium is given by:

$$
\begin{aligned}
& \frac{d \Pi}{d X_{1}}=-\sum_{j=1}^{n} A_{j} l_{j}\left(\frac{\sigma_{j}-\zeta}{E_{s h}}+\varepsilon_{j, 0}\right) s_{j, 1}=0, \\
& \frac{d \Pi}{d X_{2}}=-\sum_{j=1}^{n} A_{j} l_{j}\left(\frac{\sigma_{j}-\zeta}{E_{s h}}+\varepsilon_{j, 0}\right) s_{j, 2}=0, \\
& \ldots, \\
& \frac{d \Pi}{d X_{m}}=-\sum_{j=1}^{n} A_{j} l_{j}\left(\frac{\sigma_{j}-\zeta}{E_{s h}}+\varepsilon_{j, 0}\right) s_{j, m}=0 .
\end{aligned}
$$

This is identical to $-\partial B / \partial X_{i}$ for structures built of inelastic materials, where $\mathrm{B}$ is the complementary strain energy. This was first presented by Engesser (1889).

The equivalent equation for materials with an elastic response, i.e. $\varepsilon_{j}=\sigma_{j} / E_{1} \leq \varepsilon_{y}$ is given by:

$$
\begin{aligned}
& \frac{d \Pi}{d X_{1}}=-\sum_{j=1}^{n} A_{j} l_{j}\left(\frac{\sigma_{j}}{E_{1}}+\varepsilon_{j, 0}\right) s_{j, 1}=0, \\
& \frac{d \Pi}{d X_{2}}=-\sum_{j=1}^{n} A_{j} l_{j}\left(\frac{\sigma_{j}}{E_{1}}+\varepsilon_{j, 0}\right) s_{j, 2}=0, \\
& \ldots ., \\
& \frac{d \Pi}{d X_{m}}=-\sum_{j=1}^{n} A_{j} l_{j}\left(\frac{\sigma_{j}}{E_{1}}+\varepsilon_{j, 0}\right) s_{j, m}=0 .
\end{aligned}
$$

At the derivation of this expressions $\Delta w=0$. This appears to be identical to $-\partial \mathrm{A} / \partial X_{i}$ for structures built of elastic materials, where $\mathrm{A}$ is the strain energy. This was originally presented by Castigliano (1879).

Typically, only a part of the bars will be above the yield point. This means that Eqns (16) must be applied to the bars, which is not subjected to yielding, and Eqns (15) 
must be applied to the bars subjected to yielding. Since the rewriting of the external load is identical for the two cases, this separation into the two groups takes place without further rewritings.

By comparing Eqns (15) and (16) it is seen that by expressing these as a function of the strains, Eqns (15) and (16) can be expressed by the same set of equations:

$$
\left.\begin{array}{l}
\frac{d \Pi}{d X_{1}}=-\sum_{j=1}^{n} A_{j} l_{j} \varepsilon_{j} s_{j, 1}=0, \\
\frac{d \Pi}{d X_{2}}=-\sum_{j=1}^{n} A_{j} l_{j} \varepsilon_{j} s_{j, 2}=0, \\
\ldots ., \\
\frac{d \Pi}{d X_{m}}=-\sum_{j=1}^{n} A_{j} l_{j} \varepsilon_{j} s_{j, m}=0
\end{array}\right\} \varepsilon_{j}= \begin{cases}\frac{\sigma_{j}}{E_{1}}+\varepsilon_{j, 0} & \sigma_{j} \leq f_{y} \\
\frac{\sigma_{j}-\zeta}{E_{s h}}+\varepsilon_{j, 0} & \sigma_{j}>f_{y} .\end{cases}
$$

By returning to the example with the two times indeterminate truss structure, Eqn (17) can directly be used:

$$
\frac{d \Pi}{d X_{i}}=-\sum_{j=1}^{4} A l_{j} \varepsilon_{j} s_{j, i}=0, \quad \varepsilon_{i}=\left\{\begin{array}{ll}
\frac{\sigma_{j}}{E_{1}}+\varepsilon_{j, 0} & \varepsilon_{j} \leq \varepsilon_{y} \\
\frac{\sigma_{j}-\zeta}{E_{s h}}+\varepsilon_{j, 0} & \varepsilon_{j}>\varepsilon_{y}
\end{array} .\right.
$$

In this example there are two equations with two unknowns, corresponding to $i=1$, respectively $i=2$. $X_{1} A / P$ and $X_{2} A / P$ are shown as functions of $P / P_{u}$ in Figure 5. $P_{u}$ is defined as the load at which yielding initiated in the bar 1 (yielding in bar 3 and bar 4 has initiated at lover load levels), i.e. $\sigma_{1}=f_{y}$. The following values have been used in the example: $l_{1}=4000 \mathrm{~mm}, A=100 \mathrm{~mm}^{2}$, $f_{y}=500 \mathrm{MPa}, E_{1}=200000 \mathrm{MPa}, E_{s h}=0.05 \cdot E_{1}$.

At $P / P_{u}=0.77$ yielding in bar 4 is reached. At $P / P_{u}=0.98$ yielding in bar 3 is reached. Up to $P / P_{u}=$ 0.77 there is no energy loss. In the range $P / P_{u}=[0.77$, 0.98 ] energy is lost in bar 4. At higher values than $P / P_{u}=$ 0.98 energy is lost in both bar 3 and bar 4 . The displayed

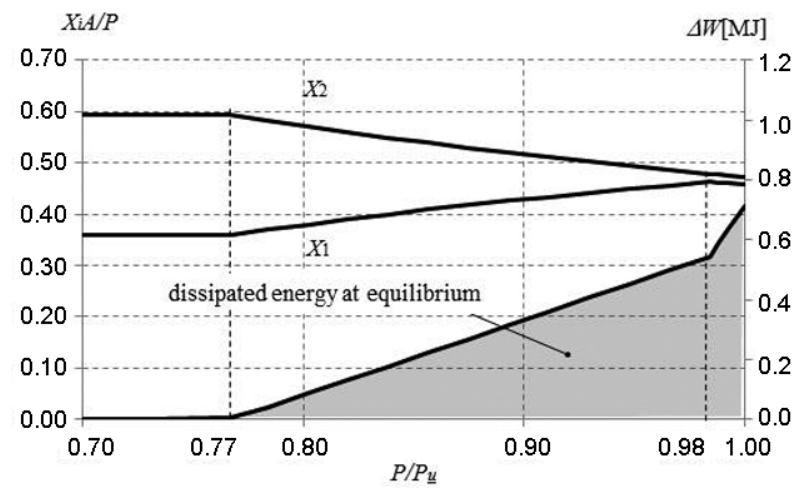

Fig. 5. Relative values of the dissipated energy at equilibrium and the redundant $X_{1}$ and $X_{2}$ as function of the load level for the truss structure shown in Figure 3 value of the lost energy is equivalent to the lost energy at equilibrium at the respective load levels corresponding to $\Pi_{\mathrm{B}}{ }^{\prime}-\Pi_{\mathrm{B}}$ in Figure $2 \mathrm{~b}$. The values of $X_{1}$ and $X_{2}$, changes as a result of the increasing dissipated energy. The maximum strain occurs in bar 4 , at $P / P_{u}=1$, and is given by $\varepsilon_{\max , 4}=1.77 \cdot \varepsilon_{y}$.

\subsection{Varying internal stresses}

Finding the state of equilibrium for structures of inelastic materials by determining the stationary state of the potential energy is a generally applicable concept. For structures constructed of elements with varying internal stresses, the concept is the same. The concepts of setting up expressions for $\Pi_{P}, \Pi_{\text {elastic }}$ and $\Delta w$ are basically identical to the case with elements with constant internal stresses. This is illustrated by examining a frame structure. For a frame structure the potential energy is also determined by Eqn (2). In Figure 6a a bilinear relation between the moment and the curvature is shown. The diagram can be subdivided into four different parts. The different parts are denoted $\mathrm{A}, \mathrm{B}_{1}, \mathrm{~B}_{2}$ and $\mathrm{C}$. The section under consideration has the bending moment $M_{j}$ and the corresponding curvature $\kappa_{j}$. For beams, or parts of beams, with inelastic response, the elastic potential energy is represented by A. The dissipated energy is represented by C. The loss of potential energy derived from the external loadings can, by use of the principles of virtual work, be expressed by the negative value of: $A+B_{1}+B_{2}+C$. This is analogous to the rewriting of Eqn (11). According to Eqn (2) the potential energy is thus expressed by the negative value of $\mathrm{B}_{1}+\mathrm{B}_{2}$. According to Figure $6 \mathrm{~b}$ the potential energy can, in the elastic case, be expressed by the negative value of $\mathrm{B}$.

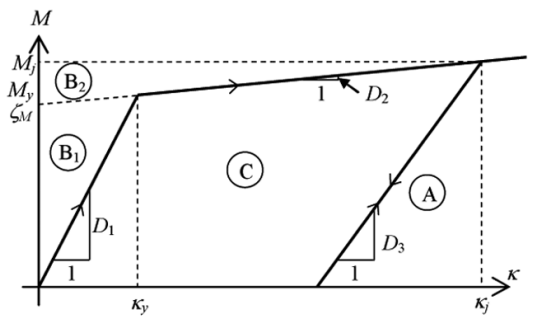

a)

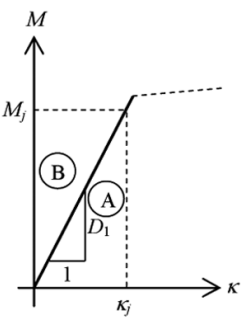

b)
Fig. 6. a) inelastic response; b) elastic response

The potential energy for an incremental part $d x$ of a beam:

$$
d \Pi= \begin{cases}-\frac{1}{2} M_{j} \cdot \kappa_{j} d x, \quad \kappa_{j}=\frac{M_{j}}{D_{1}} & M_{j} \leq M_{y} \\ -\left(\frac{1}{2} \zeta_{M} \cdot \kappa_{y}+\frac{1}{2}\left(M_{j}-\zeta_{M}\right) \cdot \kappa_{j}\right) d x, & M_{j}>M_{y} .\end{cases}
$$

This expression can be seen to be analogue to Eqn (14). Due to the variation of the internal forces the potential energy must be found by integration: 


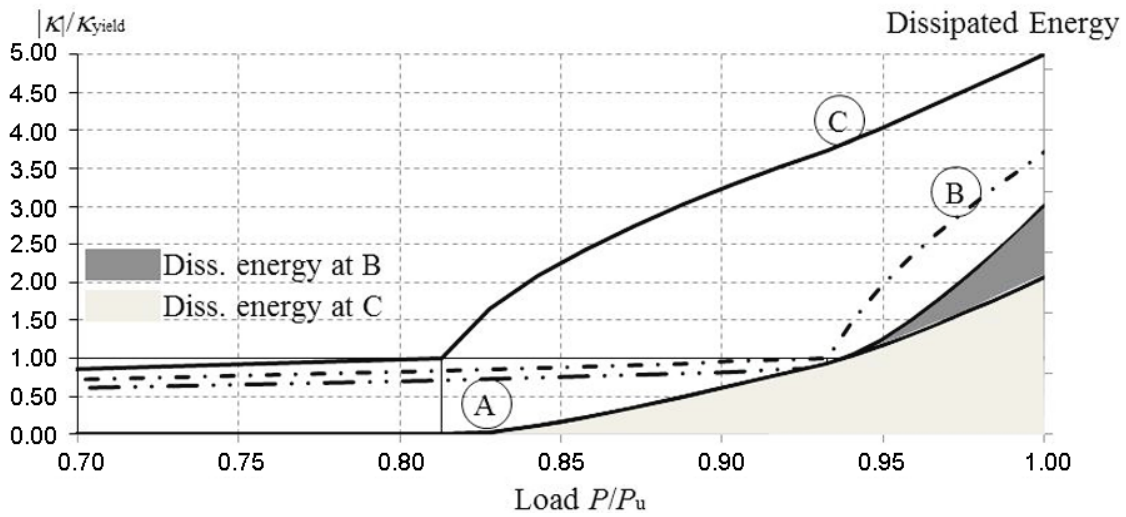

Fig. 7. Relative curvatures in points A, B and C and dissipated energy as function of the load level

$$
\begin{gathered}
\Pi=-\int_{\text {inelastic parts }}\left(\frac{1}{2} \zeta_{M} \cdot \kappa_{y}+\frac{1}{2} \frac{\left(M_{j}-\zeta_{M}\right)^{2}}{D_{2}}\right) d x- \\
\int_{\text {elastic parts }} \frac{1}{2} \frac{M_{j}^{2}}{D_{1}} d x .
\end{gathered}
$$

The moment, $M_{j}$ can be expressed as linear functions of the redundant variables, $X_{1}, X_{2}, \ldots$ The stationary state of the potential energy is found for $d \Pi / d X_{1}=0$, $d \Pi / d X_{2}=0$ and so forth.

The two times statically indeterminate frame structure shown in Figure $1 \mathrm{~b}$ is considered. In Figure 7 the relative curvature at point $\mathrm{A}, \mathrm{B}$ and $\mathrm{C}$ as well as the dissipated energy are shown as function of the load level.

The ratio between $D_{2}$ and $D_{1}$ in the example is given by $D_{2} / D_{1}=0.02$. Yielding initiates at point $\mathrm{C}$ at a load of $P=0.81 P_{u}$ and initiates at point B at a load of $P=0.93 P_{u}$. It is seen that the necessary capacity with respect to the curvature at point $\mathrm{C}$ is 5.0 times the curvature corresponding to yielding in order to achieve the full capacity.

\section{Conclusions}

It is shown that the stable state of equilibrium can be determined by minimizing the potential energy for structures constructed of inelastic materials. Thereby a physically based description of achieving equilibrium has been established.

The change in potential energy from the external loads is independent of the response of the materials. The change of the potential energy in the materials is the increase of the elastic energy. However, the dissipated energy must simultaneously be subtracted the potential energy. Changes of strains in the structure, from non-static conditions, such as thermal deformations and shrinkage, as well as plastic strains from previous load scenarios, will also change the potential energy. Thus the method is also capable of taken effects from thermal deformations and shrinkages into account. The number of equations equals the number of redundant variables of the structure. For structures with constant stress level in the individual members it turns out that the method involves solving a number of equations which are all functions of the strains.

An important application of the method is to determine the necessary ductility for a given statically indeterminate structure. And thus the ductility required achieving the full capacity of a given structure.

There is thus established a physically based method for the determination of equilibrium for structures constructed of inelastic materials. As a direct result of the determination of the equilibrium is also determined the necessary capacity with respect to the ductility.

\section{References}

Balázs, G. L.; Bisch, P.; Borosnyói, A.; Burdet, O.; Burns, C.; Ceroni, F.; Cervenka, V.; Chiorino, M. A.; Debernardi, P.; Eckfeldt, L.; El-Badry, M.; Fehling, E.; Foster, S. J.; Ghali, A.; Gribniak, V.; Guiglia, M.; Kaklauskas, G.; Lark, R. J.; Lenkei, P.; Lorrain, M.; Marí, A.; Ozbolt, J.; Pecce, M.; Caldentey, A. P.; Taliano, M.; Tkalcic, D.; Torrenti, J. M.; Torres, L.; Toutlemonde, F.; Ueda, T.; Vitek, J. L.; Vráblík, L. 2013. Design for SLS according to fib Model Code 2010, Structural Concrete 14(2): 99-123. http://dx.doi.org/10.1002/suco.201200060

Beedle, L. S. 1957. Plastic strength of steel frames, in Proceedings of Third National Construction Industry Conference, 11-12 December 1957. $16 \mathrm{p}$.

Berman, J. W.; Bruneau, M. 2003. Plastic analysis and design of steel plate shear walls, Journal of Structural Engineering 129(11): 1148-1456.

Bruneau, M.; Uang, C. M.; Sabelli, R. 2011. Ductile design of steel structures. $2^{\text {nd }}$ ed. McGraw Hill.

Castigliano, C. A. 1879. Théorie de l'équilibre des systèmes élastiques, et ses applications. A. F. Negro.

Castigliano, C. A.; Oravas, G.; Andrews, E. S. 1966. Theory of equilibrium of elastic systems and its applications. New York: Dover Publications, Inc.

Drucker, D. C.; Greenberg, H. J.; Prager, W. 1952. Extended limit design theorems for continuous media, Quarterly of Applied Mathematics 9(4): 381-389.

Einpaul, J.; Ruiz, M. F.; Muttoni, A. 2015. Influence of moment redistribution and compressive membrane action on punching strength of flat slabs, Engineering Structures 86: 43-57. http://dx.doi.org/10.1016/j.engstruct.2014.12.032 
Engesser, F. 1889. Ueber statisch unbestimmte Träger bei beliebigem Formänderungs-Gesetze und über den Satz von der kleinsten Ergänzungsarbeit. Zeitschrift Architekten- und Ingenieur-Vereins zu Hannover. Band XXXV. Heft 8.

Feynman, R. P.; Leighton, R. B.; Sand, M. 2011. The Feynman lectures on physics. Volume I. Basic Books.

Fisker J.; Hagsten, L. G. 2016. Mechanical model for the shear capacity of R/C beams without stirrups: A proposal based on limit analysis, Engineering Structures 115: 220-231. http://dx.doi.org/10.1016/j.engstruct.2016.02.035

Helmholtz, H. 1847. Ueber die Erhaltung der Kraft. Berlin: Druck und Verlag von G. Reimer.

Lee, S. C.; Cho, J. Y.; Vecchio, F. J. 2011. Model for post-yield tension stiffening and rebar rupture in concrete members, Engineering Structures 33(5): 1723-1733.

http://dx.doi.org/10.1016/j.engstruct.2011.02.009

Marti, P. 2013. Theory of structures: fundamentals, framed structures, plates and shells. Wiley. http://dx.doi.org/10.1002/9783433602638

Montuori, R.; Nastri, E.; Piluso, V. 2014. Rigid-plastic analysis and moment-shear interaction for hierarchy criteria of inverted Y EB-Frames, Journal of Constructional Steel Research 95: 71-80. http://dx.doi.org/10.1016/j.jcsr.2013.11.013

Montuori, R.; Nastri, E.; Piluso, V. 2015. Advances in theory of plastic mechanism control: closed form solution for MRFrames, Earthquake Engineering \& Structural Dynamics 44(7): 1035-1054. http://dx.doi.org/10.1002/eqe.2498

Muttoni A.; Ruiz, M. F.; Niketic, F. 2015. Design versus assessment of concrete structures using stress fields and strutand-tie models, ACI Structural Journal 112(5): 605-616. http://dx.doi.org/10.14359/51687710

Nastri, E.; Montuori, R.; Piluso, V. 2015. Seismic design of MRF-EBF dual systems with vertical links: EC8 vs plastic design, Journal of Earthquake Engineering 19(3): 480504. http://dx.doi.org/10.1080/13632469.2014.978917
Nethercot, D. A. 2000. Frame structures: global performance, static and stability behaviour: General report, Journal of Constructional Steel Research 55(1-3): 109-124. http://dx.doi.org/10.1016/S0143-974X(99)00080-2

Nielsen, M. P.; Hoang, L. C. 2011. Limit analysis and concrete plasticity. $3^{\text {rd }}$ ed. CRC Press.

Peng, J. L.; Wu, C. W.; Chan, S. L.; Huang, C.-H. 2013. Experimental and numerical studies of practical system scaffolds, Journal of Constructional Steel Research 91: 64-75. http://dx.doi.org/10.1016/j.jcsr.2013.07.028

Qu, B.; Bruneau, M. 2010. Capacity design of intermediate horizontal boundary elements of steel plate shear walls, Journal of Structural Engineering 136(6): 665-675. http://dx.doi.org/10.1061/(asce)st.1943-541x.0000167

Ruiz, M. F.; Mirzaei, Y.; Muttoni, A. 2013. Post-punching behavior of flat slabs, ACI Structural Journal 110(5): 801812.

Sahoo, D. R.; Chao, S. H. 2010. Performance-based plastic design method for buckling-restrained braced frames, Engineering Structures 32(9): 2950-2958. http://dx.doi.org/10.1016/j.engstruct.2010.05.014

Santos, H. 2011. Complementary-energy methods for geometrically non-linear structural models: An overview and recent developments in the analysis of frames, Archives of Computational Methods in Engineering 18(1): 405-440. http://dx.doi.org/10.1007/s11831-011-9065-6

Simões, J. T.; Faria, D. M. V.; Ruiz, M. F.; Muttoni, A. 2016. Strength of reinforced concrete footings without transverse reinforcement according to limit analysis, Engineering Structures 112: 146-161. http://dx.doi.org/10.1016/j.engstruct.2016.01.010

Wongpakdee, N.; Leelataviwat, S.; Goel, S. C.; Liao W.-C. 2014. Performance-based design and collapse evaluation of buckling restrained knee braced truss moment frames, Engineering Structures 60: 23-31. http://dx.doi.org/10.1016/j.engstruct.2013.12.014

Lars German HAGSTEN. Born in 1969. Is responsible for the graduate programme in Civil and Architectural Engineering, offered by Aarhus University, and group leader of the research group in Structural Analysis and Concrete Structures. Lars German Hagsten graduated as a Master of Science from the Technical University of Denmark in 1995, and completed his PhD as an industrial researcher in 2000. He has ten years of experience from Ramboll, Alectia working as a consultant engineer and specialist leader of brickwork constructions, and later from COWI A/S, working with design- and project engineering of mainly concrete- and steel constructions, as well as acting as specialist leader. 